The Astronomical Journal, accepted

Preprint typeset using $\mathrm{IAT}_{\mathrm{E}} \mathrm{X}$ style emulateapj v. 11/10/09

\title{
STARS AND IONIZED GAS IN S0 GALAXY NGC 7743: AN INCLINED LARGE-SCALE GASEOUS DISK.
}

IVAN YU. KATKOV

Sternberg Astronomical Institute, Moscow, 119992 Russia

Alexei V. Moiseev

Special Astrophysical Observatory, Russian Academy of Sciences, Nizhnii Arkhyz, Karachaevo-Cherkesskaya Republic, 369167 Russia

\author{
Olga K. Sil'ChenKo \\ Sternberg Astronomical Institute, Moscow, 119992 Russia \\ and Isaac Newton Institute of Chile, Moscow Branch \\ The Astronomical Journal, accepted
}

\begin{abstract}
We used deep long-slit spectra and integral-field spectral data to study the stars and ionized gas kinematics and stellar population properties in the lenticular barred galaxy NGC 7743. We have shown that ionized gas at the distances larger than $1.5 \mathrm{kpc}$ from the nucleus settles in the disk which is significantly inclined to the stellar disk of the galaxy. Making different assumptions about the geometry of the disks and involving different sets of emission lines into the fitting, under the assumption of thin flat disk circular rotation, we obtain the full possible range of angle between the disks to be $34 \pm 9^{\circ}$ or $77 \pm 9^{\circ}$. The most probable origin of the inclined disk is the external gas accretion from a satellite, orbiting the host galaxy with a corresponding angular momentum direction. The published data on the HI distribution around NGC 7743 suggest that the galaxy has a gas-rich environment. The emission-line ratio diagrams imply the domination of shock waves in the ionization state of the gaseous disk, whereas the contribution of photoionization by recent star formation seems to be negligible. In some parts of the disk a difference between the velocities of the gas emitting in the forbidden lines and in the Balmer lines is detected. It may be caused by the fact that the inclined disk is mainly shock-excited, whereas some fraction of the Balmer-line emission is produced by a small amount of gas excited by young stars in the main stellar disk of NGC 7743. In the circumnuclear region $(R<200 \mathrm{pc})$ some evidences of the AGN jet interaction with an ambient interstellar medium were found.

Subject headings: galaxies: elliptical and lenticular — galaxies: ISM — galaxies: kinematics and dynamics — galaxies: interactions — galaxies: individual: NGC 7743.
\end{abstract}

\section{INTRODUCTION}

Interactions play an important role in the galaxy evolution. Even a minor merger with the mass ratio less than $1 / 5-1 / 10$ which does not disturb the overall structure of the galaxy disk, may cause a gas concentration in its central region, triggering the nuclear activity or a nuclear starburst. According to the hierarchical paradigm, such events happened many times during a galaxy lifetime, however, it is difficult to detect minor merging footprints, even the recent ones, against the background of a high surface brightness galaxy. Deep images, revealing low-contrast tidal features (see, for example Martínez-Delgado et al. 2010; Smirnova et al. 2010), or detailed studies of stellar populations and internal kinematics of the galaxy are required.

The consequences of a minor merger may be quite diverse. Own gas of a larger galaxy may be disturbed by a satellite intrusion, hence it may inflow into the center, resulting in a strong gas compression in the nucleus and a subsequent nuclear star formation burst. A rather young current age of the nuclear star population inside

katkov.ivan@gmail.com

moisav@gmail.com

olga@sai.msu.su an older bulge may be the signature of such event. If the initial direction of the orbital angular momentum of the satellite significantly differed from the main galaxy disk rotation momentum, then we may now have a situation when some fraction of stars or gas clouds in the galaxy are rotating on orbits tilted (or orthogonal) to the main galactic disk, or even counter-rotating within it. Longslit and integral-field spectral observations gave a number of evidences for the kinematical misalignments in circumnuclear regions of early-type disk galaxies including the cases of kinematically decoupled cores. Numerous examples and detailed discussions and references can be found, for instance, in the papers by Afanasiev et al. (1989); Corsini et al. (2003); Moiseev et al. (2004); Sarzi et al. (2006); Coccato et al. (2007). Unfortunately, observational evidences of large-scale (beyond one kiloparsec central region) kinematically decoupled subsystems are still quite rare (see Sil'chenko et al. 2009, and references therein). Therefore, every new example of the similarly peculiar objects is interesting.

In the present paper, we demonstrate that the main fraction of the ionized gas in NGC 7743 rotates on the orbits, considerably inclined to the main stellar disk of the galaxy, what may be a result of external gas accretion or tidal destruction of a gas-rich small companion. The 


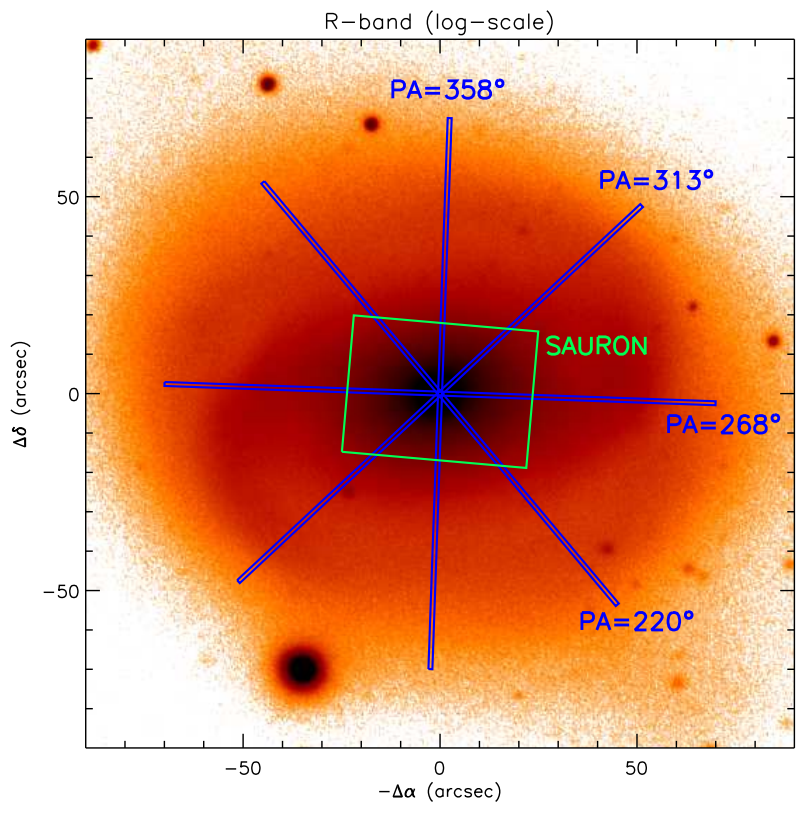

FIG. 1.- The positions of the SCORPIO slits and the SAURON mosaic field of view are overlapped onto the $R$-band image of the galaxy NGC 7743 from Moiseev et al. (2004).

paper is organized as follows: Section 2 gives an overview of the literature on the previous studies of the galaxy; Section 3 describes the spectroscopic observations and data reduction process; in Section 4 the properties of the stellar population (kinematics, age, and metallicity) are considered; Section 5 contains the study of gas kinematics and ionization state; Section 6 includes an overall discussion of the structure and kinematics of NGC 7743.

\section{NGC 7743: WHAT WAS KNOWN BEFORE?}

NGC 7743 is a barred early-type galaxy (NED morphological type $(\mathrm{R}) \mathrm{SB}^{+}(\mathrm{s})$ ) with a total blue luminosity of $M_{B}=-19.4$ (according to the HyperLeda databas $\square$ ). Following Jensen et al. (2003), we adopt the distance to the galaxy to be $19.2 \mathrm{Mpc}$, that corresponds to a linear scale of $93 \mathrm{pc}^{\operatorname{arcsec}^{-1}}$.

A smooth two-armed spiral structure without any traces of star formation dominates in that optical images of the galaxy. According to Ho et al. (1997) the galaxy has an active nucleus of the Sy2 type. Radio observations reveal a compact (under the beam $=1^{\prime \prime}-5^{\prime \prime}$ ) non-thermal source in the nucleus (Nagar et al. 1993: Ho \& Ulvestad 2001). However, the nuclear activity is not very high. Alonso-Herrero et al. (2000) using their spectral observations classified NGC 7743 as a 'lowluminosity AGN' and noted that the optical emission line ratios correspond to the boundary case between Sy2 and LINER activity types. The X-ray observations by Terashima et al. (2002) also suggest a relatively low activity of the NGC 7743 nucleus, compared with other Seyfert galaxies from their sample.

The HST imaging reveals a complex structure of the circumnuclear region $(R<300-500$ pc) where emis-

\footnotetext{
1 http://leda.univ-lyon1.fr
}

sion knots and several curved dust lanes are observed (Regan \& Mulchaev 1999). Martini et al. (2003) described this structure as a loosely wound circumnuclear spiral. Moiseev et al. 2004) used integral-field spectroscopy to study the inner region morphology and kinematics. They suggested that a turn of the innermost isophotes relates with the circumnuclear dust spiral, rather than with a triaxial bulge as it was claimed earlier. Despite the relatively low intensity of emission lines, they succeeded to obtain some conclusions about the ionized gas kinematics. In the region of $R<2^{\prime \prime}-4^{\prime \prime}$ (190-370 pc) the gas motions agree in general with the stellar disk rotation, but non-circular ionized gas motions were also detected locally to the south from the nucleus. Moiseev et al. (2004) have as well supposed that the inner part of the galaxy disk can be tilted with respect to the outer disk.

Radio observations by Duprie \& Schneider (1996) demonstrated a very low content of neutral gas (near their detection limit) for the NGC 7743 disk through the $\sim 3^{\prime}$ beam, whereas two separate HI clouds were discovered in the tight neighborhood of the galaxy having the masses of $M_{H I}=6.4 \times 10^{7} M_{\odot}$ and $4.5 \times 10^{8} M_{\odot}$. Their systemic velocities are $1610 \mathrm{~km} \mathrm{~s}^{-1}$ and $1509 \mathrm{kms}^{-1}$, respectively, that is close to the NGC 7743 systemic velocity which is $1710 \mathrm{kms}^{-1}$ (HyperLeda). According to the NED database these clouds are associated with the galaxies KUG 2341+097 and LSBC F750-04, with projected distances of 8.3 (46 kpc) and 11.0 (61 kpc) from NGC 7743. The absolute magnitude of the brighter galaxy (KUG $2341+097$ ) is about $M_{B}=-16$. Therefore NGC 7743 is surrounded by the satellites which altogether contain the neutral hydrogen amount by one order larger compared to the disk of the main galaxy. At the same time Maiolino et al. (1997) from their radio observations with a beam $=55^{\prime \prime}$ detected the $\mathrm{CO}$ emission, corresponding to the total mass of the molecular gas in the galaxy disk of about $1.3 \times 10^{8} M_{\odot}$, which is comparable with the HI mass in the local environment of NGC 7743.

\section{SPECTRAL OBSERVATIONS AND DATA REDUCTION}

In our study of NGC 7743, we have used two types of spectral data. Long-slit spectroscopy with exposures long enough allows to reach very outer parts of the galaxy disk; however, one-dimensional character of the long-slit data restricts consideration of any azimuthal variations. Integral-field spectroscopy (so called '3D spectroscopy') provides two-dimensional mapping of the kinematical and stellar population characteristics but only for the central part of the galaxy due to the limited field-of-view.

\subsection{SCORPIO long-slit observations.}

The spectral observations were made at the prime focus of the SAO RAS 6-m telescope with the multimode focal reducer SCORPIO (Afanasiev \& Moiseev 2005). The slit had 6.1 in length and $1^{\prime \prime}$ in width. The $2048 \times 2048 \mathrm{EEV}$ 42-40 CCD provided the scale of $0.35 \operatorname{arcsec} \mathrm{px}^{-1}$ along the slit. The log of observations is given in Table 1, the slits positions onto the galaxy image are shown in Fig. 1.

The starting observations in 2007 were performed in order to study the stellar kinematics (P.I. of the proposal was Anatoly Zasov), therefore, the 'green' spectral range of $4800-5550 \AA$ was chosen. It contains strong stellar 
TABLE 1

LOG OF THE OBSERVATIONS

\begin{tabular}{|c|c|c|c|c|c|c|}
\hline Instrument & Date & $P A(\operatorname{deg})$ & Sp. range $(\AA)$ & Sp. resolution $(\AA)$ & Exp. time (s) & Seeing $\left({ }^{\prime \prime}\right)$ \\
\hline \multirow[t]{6}{*}{ SCORPIO } & 2007 Sep 21 & 268 & $4800-5550$ & 2.5 & $9 \times 1200$ & 1.9 \\
\hline & 2009 Jan 22 & 268 & $6100-7100$ & 3.1 & $2 \times 500$ & 2.4 \\
\hline & 2007 Sep 21 & 358 & $4800-5550$ & 2.5 & $9 \times 1200$ & 1.8 \\
\hline & 2009 Jan 22 & 358 & $6100-7100$ & 3.1 & $2 \times 400$ & 3.0 \\
\hline & 2008 Dec 19 & 313 & $4800-5550$ & 2.5 & $8 \times 1200$ & 1.2 \\
\hline & 2009 Oct 15 & 220 & $6100-7100$ & 3.1 & $10 \times 1200$ & 1.6 \\
\hline \multirow[t]{2}{*}{ SAURON } & 2007 Aug 14 & 355 (Pos. 1) & $4800-5400$ & 4.8 & $2 \times 1800$ & 2 \\
\hline & 2007 Aug 14 & 355 (Pos. 2) & $4800-5400$ & 4.8 & $2 \times 1800$ & 2 \\
\hline
\end{tabular}

absorption line features as well as the ionized gas emission lines $-\mathrm{H} \beta$ and $[\mathrm{OIII}] \lambda \lambda 4959,5007$. After the preliminary analysis of these data a difference in the kinematics of gas and stars was found, and we carried out additional observations in the 'red' spectral domain of 6100-7100 $\AA$ including the brighter emission lines - $\mathrm{H} \alpha$, $[\mathrm{N} \mathrm{II}] \lambda \lambda 6548,6583$, and [S II] $\lambda \lambda 6716,6731$.

The spectra were reduced by the standard way using our IDL-based software (see, for instance, Zasov et al. 2008). For the night sky spectrum subtraction, we applied an advanced technique taking into account the variations of the Line Spread Function (LSF) along the slit (Katkov \& Chilingarian 2011). The model of the LSF was derived from the twilight sky spectra observed the same nights as the galaxy. These spectra were binned by 10 pixels along the slit, the wavelength range was divided into six segments. The LSF parameters were estimated over every segment by fitting the observed spectrum by a broadened high-resolution spectrum of the Sun using the ULySS software2 (Koleva et al. 2008b, 2009) adapted for the SCORPIO data.

The further data analysis included following steps:

- Adaptive binning along the slit - we summed spectra in order to achieve the minimal value of signalto-noise ratio $S / N=20-30$.

- Masking the emission line regions and fitting the binned galaxy spectra with a simple stellar population (SSP) models PEGASE.HR (Le Borgne et al. 2004) convolved with a parametric line-of-sight velocity distribution (LOSVD) using ULySS software. The resulting stellar physical parameters are the line-of-sight velocity $-v$, velocity dispersion $-\sigma$, higher-order Gauss-Hermite moments $h_{3}$, $h_{4}$; and the SSP-equivalent luminosity-weighted parameters: the age $T$ and metallicity $[\mathrm{Z} / \mathrm{H}]$. The stellar population models were constructed with the local star spectra library, therefore, the element abundance ratios, $[\mathrm{Mg} / \mathrm{Fe}]$ in particular, cannot be free variables. While we deal with a rather high model metallicity, $[\mathrm{Fe} / \mathrm{H}]>-0.4$, the model alphaelement-to-iron ratio is necessarily close to the solar one due to the fixed chemical properties of the stars in the Sun' neighborhood. For a more detailed description see Koleva et al. (2008b, 2009).

- The subtraction of the stellar contribution from non-binned spectra using the model stellar spectra evaluated in every bin at the previous step, and normalized here by a polynomial approximation of

\footnotetext{
2 http://ulyss.univ-lyon1.fr/
}

the surface brightness distribution. The result is a non-binned ionized gas spectrum.

- Adaptive binning of the emission lines spectra. The bins were formed under the condition that $S / N>$ 10 for the targeted emission line.

- The emission lines fitting using the Gaussians convolved with the LSF. For the doublets of [OIII], $[\mathrm{NII}]$, and $[\mathrm{SII}]$ the restframe separation between components, their widths and intensity ratios (except the sulfur lines) were fixed.

It should be noted that the cross-sections $P A=268^{\circ}$ and $358^{\circ}$ in the 'red' spectral range were observed with relatively low exposure times, and emission lines measurements in these spectra gave no any additional information about the ionized gas kinematics with respect to the deep spectra in the 'green' spectral domain. Therefore we have used the measurements of the emission lines $\mathrm{H} \alpha,[\mathrm{NII}]$ and $[\mathrm{SII}]$ in these $P A s$ only to derive the ionization state diagrams. On the contrary, in $P A=220^{\circ}$ (the galaxy minor axis) we took very deep 'red' spectra, where the ionized gas emission lines were detected at large distances from the nucleus. At the same time the estimations of the stellar population parameters in the 'red' domain are uncertain, opposite to the 'green' spectra.

The kinematical profiles for the stellar and gaseous components, the emission lines ratios and radial profiles of the stellar population parameters are shown in Fig. 2.

\subsection{SAURON integral-field spectroscopy.}

We also involved the data from the 4.2-m William Herschel Telescope integral-field spectrograph SAURON (Bacon et al. 2001) obtained in the framework of the ATLAS3D survey (Cappellari et al. 2011). The raw data have been retrieved by us from the open Isaac Newton Group Archive, which is maintained as a part of the CASU Astronomical Data Centre at the Institute of Astronomy, Cambridge. NGC 7743 was observed in two positions of the SAURON lenslet array $33^{\prime \prime} \times 41^{\prime \prime}$ in size centered onto the opposite sides relative to the galactic nucleus. The resulting central part of the galaxy observed was $35^{\prime \prime} \times 50^{\prime \prime}$ (see Fig. 1) with 0.94 sampling. For our analysis we have used the scientific-ready spectral data cubes which have been presented earlier by Sil'chenko \& Chilingarian (2011).

The data cubes were fitted with the ULySS software package in the same manner as the long-slit data described above. The model of the LSF constructed from the twilight sky spectra was taken into account. Because of the relatively low $S / N$ ratio of the emission lines we 

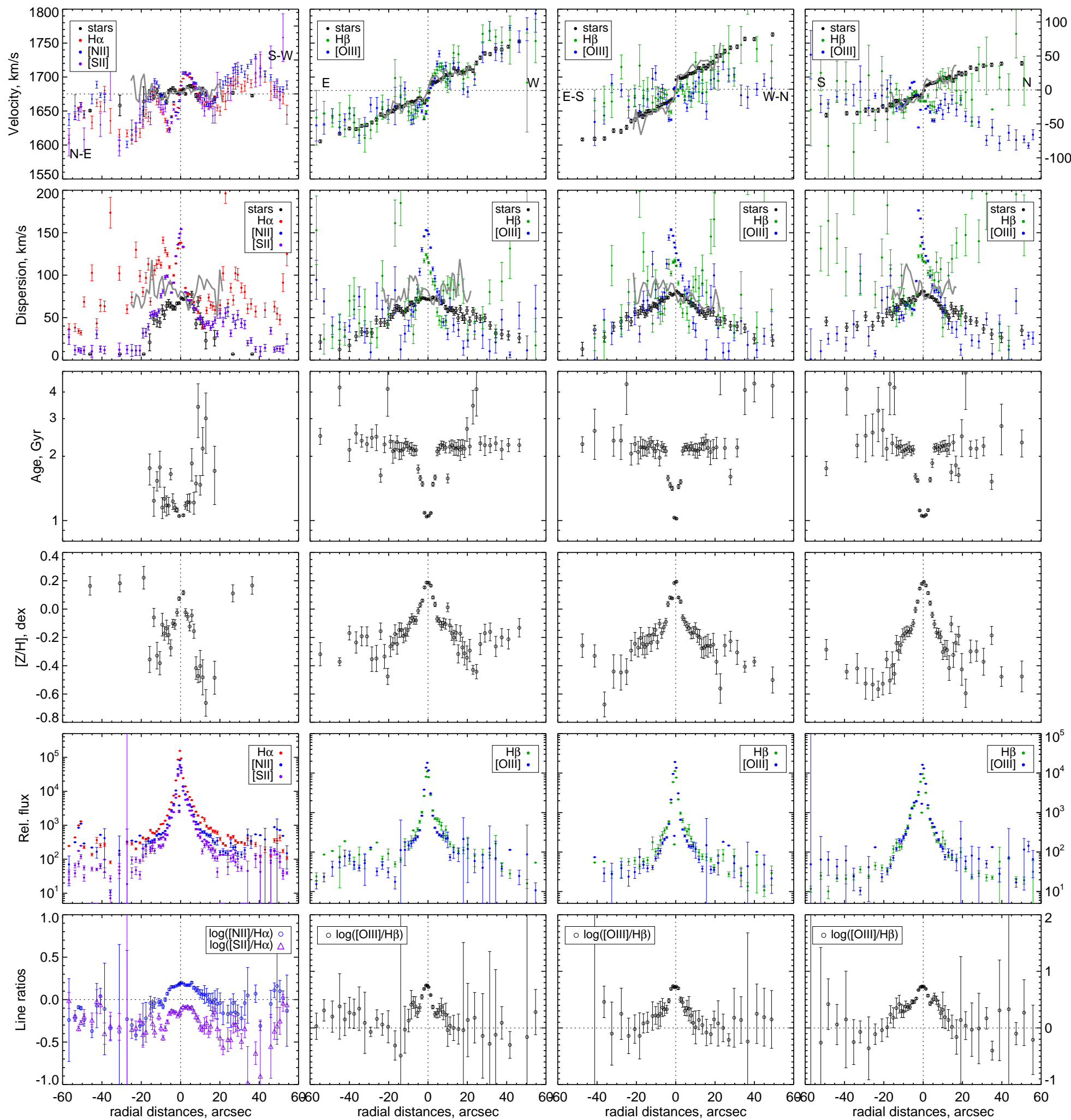

FIG. 2.- The results of the long-slit observations. Each column corresponds to the slit position angle labelled at the top. Data for $P A=220^{\circ}$ are shown for the 'red' spectral range, in other cases we present the results only for the 'green' spectral domain. From top to bottom we show the radial distributions of the line-of-sight velocity, the velocity dispersion, the age and metallicity of the stellar component, the intensity of the emission lines (in arbitrary units) and the line intensity ratios. Grey lines correspond to the pseudo slits extracted from the IFU data. 
fitted simultaneously $\mathrm{H} \beta$ and [O III] emission line profiles by assuming that their velocities are the same; the line widths and intensities were free parameters. The maps were smoothed by a Gaussian with $F W H M=1.5$ pixels. The resulting maps are presented in Fig. 3 .

\section{STELLAR CONTENT}

\subsection{Kinematics}

Radial variations of the $v$ and $\sigma$ derived from our long-slit data (Fig. 2) show a good agreement with the SAURON maps over the galaxy central region (Fig. 31). The line-of-sight velocity curve at the $P A=313^{\circ}$ (which corresponds to the kinematical major axis, see below) has a sharp small-amplitude internal maximum at the $R \approx 2^{\prime \prime}(190 \mathrm{pc})$. At larger radii the rotation velocity rises continuously up to $R \approx 60^{\prime \prime}(5.5 \mathrm{kpc})$. The apparent amplitude of the stellar rotation velocity is about $160 \mathrm{~km} \mathrm{~s}^{-1}$.

We have assumed a purely Gaussian shape of the LOSVD and ignored the contribution of the $h_{3}$ and $h_{4}$ moments, because the value of the intrinsic velocity dispersion is comparable to the width of the LSF $\left(\sigma_{L S F} \approx 65 \mathrm{~km} \mathrm{~s}^{-1}\right)$. The $\sigma$ profiles along different $P A s$ are slightly different: the cross-sections along the $P A=313^{\circ}$ and $358^{\circ}$ reveal a peak of $80 \mathrm{~km} \mathrm{~s}^{-1}$ achieved in the nucleus, while along the bar $\left(P A=268^{\circ}\right)$ a central plateau has appeared. A complex surface distribution of the $\sigma$ is confirmed by the SAURON maps (Fig. 3): in the central region $\left(R<5^{\prime \prime}\right) \sigma$ is depressed with respect to the neighboring regions. Similar 'sigma-drop' features in barred galaxies may result from a circumnuclear dynamically cold young stellar disks (Wozniak et al. 2003). Koleva et al. (2008a) have as well noted that such a $\sigma$ depression can be an artifact related to the template mismatch. However in the case of NGC 7743 we propose interpretation of the $\sigma$-drop in the framework of the Wozniak et al. (2003) concept, because the presence of young stellar population in the galaxy core is confirmed by our measurements (see the next subsection). We think also that in our case we have no problems with template mismatch: the inspection of the residuals after the model spectra subtraction gives impression of the fitting quality (Fig. 4). Note that our estimate of the central velocity dispersion is slightly lower than those of other authors: HyperLeda gives $84.5 \pm 2.4 \mathrm{~km} \mathrm{~s}^{-1}$ averaged over six measurements.

The kinematical orientation parameters were determined by applying a model of thin flat circular rotation to the observed velocity fields. The model corresponds to

$$
\begin{gathered}
V_{\text {obs }}=V_{\text {sys }}+V_{\text {rot }}(R) \frac{\cos \varphi \sin i}{\sqrt{\sec ^{2} i-\cos ^{2} \varphi \tan ^{2} i}} \\
R=r \sqrt{\sec ^{2} i-\cos ^{2} \varphi \tan ^{2} i},
\end{gathered}
$$

where $R, r$ - is the radius in the galaxy plane and the radius in the plane of the sky, $i$ is the inclination of the galaxy plane. Position angle of the kinematical major axis of the stellar disk (i.e. the disk line-of-nodes) $P A_{0}$ and angle $\varphi$ are related by $\varphi=P A_{\text {slit }}-P A_{0}$. Rotation curve $V_{\text {rot }}(R)$ was determined with several fixed radial nodes and was then interpolated to all radii using spline functions. Thus, the model parameters are the rotation velocity values at fixed radial nodes and the common inclination, and the line-of-nodes position angle. We simultaneously fitted three long-slit stellar velocity profiles (the most extensive measurements being in the 'green' domain) and the SAURON velocity field. The best model has an inclination of $i_{\text {star }}=40 \pm 2^{\circ}$ and a major axis position angle of $P A_{\text {star }}=310 \pm 5^{\circ}$. The rotation curve is shown in Fig. (5 (left column). Moiseev et al. (2004) have found $P A_{\text {star }}=300-310^{\circ}$ from the circumnuclear velocity field of stars $\left(R<7^{\prime \prime}\right)$, which almost coincides with our new measurements for the whole large-scale stellar disk. It is important to note that the large-scale bar could perturb the circular velocities of stars, however we have found that these perturbations are negligible in the case of NGC 7743. First of all, it follows from the tilted-ring analysis of the SAURON velocity field (for the method description, see Moiseev et al. 2004) that radial variations of the kinematical major-axis $P A$ demonstrate very small deviations $\left(1-2^{\circ}\right)$ from the mean $P A_{\text {star }}$ (cited above) up to $R=25^{\prime \prime}(2.3 \mathrm{kpc})$. Such a picture would have been impossible if the bar significantly distorts the velocity field - we should observe a turn of the $P A$ along the radius because the bar potential contribution decreases with the distance from the center, while in the very center the rotation of the bulge dominates the line-of-sight velocity field. On the other hand, we tried to search for the orientation parameters excluding the bar, area and using only the elements with $R>30^{\prime \prime}(2.8$ $\mathrm{kpc})$. This fit returns the value of $P A_{\text {star }}$ roughly equal to the estimates given above, but the inclination cannot be confidently determined in this fit.

\subsection{Age and metallicity}

Radial variations of the stellar population parameters derived for NGC 7743 from our long-slit data - those of the SSP-equivalent age and metallicity - are given in Fig. 2 together with the other radial profiles. The stellar nucleus of NGC 7743 has appeared to be chemically and evolutionarily decoupled: the mean stellar age in the nucleus is only $1 \mathrm{Gyr}$, the metallity is 1.5 times above solar. Beyond the nucleus, metallicity drops to subsolar values, and the age stabilizes at about $2.5 \mathrm{Gyr}$. We suppose that our measurements at $R<20^{\prime \prime}-30^{\prime \prime}$ (i.e. $1.9-2.8 \mathrm{kpc}$ ) relate mainly to the bulge and bar of NGC 7743 , based on its surface brightness distribution (Erwin et al. 2008).

Earlier, we already reported the chemical and evolutionary distinction of the nucleus of NGC 7743 with our integral-field spectroscopic results for the central part of the galaxy. By obtaining the data with the Multi-Pupil Fiber Spectrograph (MPFS) of the Russian 6-m telescope, Sil'chenko (2006) has measured the Lick indices and found that the nucleus was 5 times more metal-rich, and by more than 2 Gyr younger with respect to the neighboring bulge. However, the absolute values of the ages and metallicities obtained here differ from those, obtained by Sil'chenko (2006): there $[\mathrm{Z} / \mathrm{H}]=+0.7$ was found for the nucleus and 0.0 for the bulge, and the mean age of the bulge was determined as 4 Gyr old. The emission lines were very strong in the nuclear and circumnuclear spectra, and the excitation mechanism of those was unclear, so perhaps the correction of the Lick in$\operatorname{dex} \mathrm{H} \beta$ for the emission by Sil'chenko (2006) was not completely correct; hence, the stellar population parameters for NGC 7743 in Sil'chenko (2006) may be biased. 

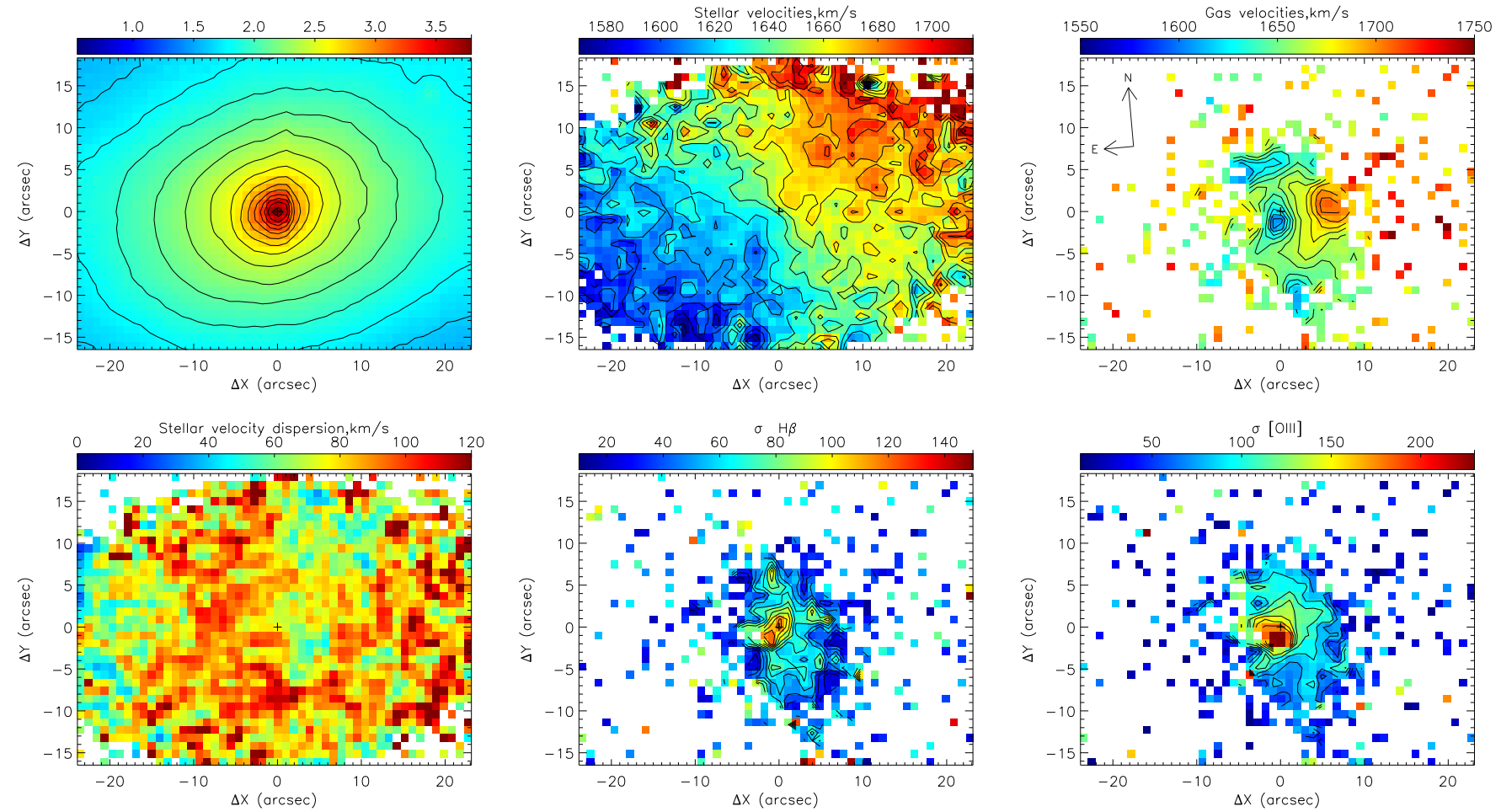

FIG. 3.- The results of the SAURON data analysis. The top row shows the continuum image, the line-of-sight velocity fields for the stars and for the ionized gas. The bottom row shows the map of the stellar component velocity dispersion and velocity dispersion maps for the lines $H \beta$ and [OIII].

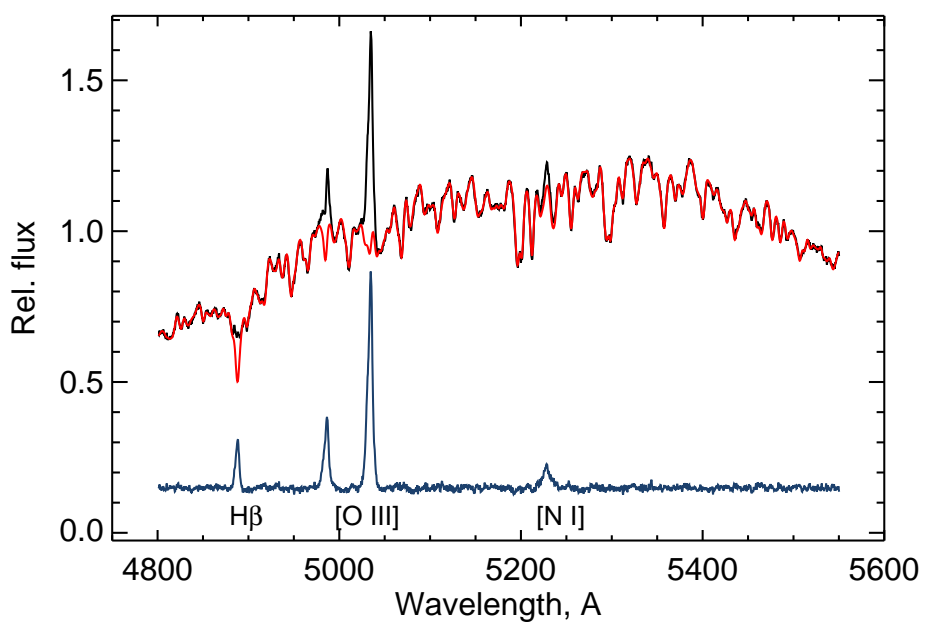

2011) while Fig. 2 implies $[\mathrm{Z} / \mathrm{H}]=+0.2$ and $T=1 \mathrm{Gyr}$ in the nucleus and $[\mathrm{Z} / \mathrm{H}]=-0.3 \pm 0.1$, and the age of 2.5 Gyr for the bulge. Therefore, the data of several spectrographs give the evidence for the young age and enhanced metallicity in the stellar nucleus of NGC 7743; we conclude that there is a signature of a recent nuclear star formation burst.

In Fig. 2 the stellar population parameter profile in $P A=268^{\circ}$, almost along the bar, is quite outstanding among the others. The semiaxis of the bar is $31^{\prime \prime}-37^{\prime \prime}(2.9-3.4 \mathrm{kpc})$, according to the estimates by Erwin (2005). At the slit orientation along the bar, unlike the other two profiles, the metallicity does not diminish toward the measurement limits, but stops its fall at the radius of about $30^{\prime \prime}$, near the end of the bar, and then even rises a little. Perhaps, here we feel the influence of so called ansae - the bright and often starforming regions at the ends of a bar which are especially frequent in barred galaxies of early morphological types (Laurikainen et al. 2007): additional star formation should increase simultaneously both a continuum surface brightness and a mean stellar metallicity.

\section{IONIZED GAS}

\subsection{Gas velocities}

the SAURON data (which are analysed here as well), by the method of direct spectrum fitting in the pixel area, that allows to exclude all the spectral regions, affected by emission. The results of the SAURON data analysis by Sil'chenko \& Chilingarian (2011) agree perfectly with the present results, derived by fitting the long-slit SCORPIO spectra: they have obtained $[\mathrm{Z} / \mathrm{H}]=+0.2$ and the age of $1.2 \mathrm{Gyr}$ for the nucleus, and $[\mathrm{Z} / \mathrm{H}]=-0.33$ and the age of 2.2 Gyr for the bulge (Sil'chenko \& Chilingarian

Gas motions in NGC 7743 are more complex than those of the stars and look decoupled in all position angles except in $P A=268^{\circ}$ ( Fig. 2). Along the $P A=358^{\circ}$ the velocity projections onto our line of sight have opposite winding senses for the gas and for the stars. By using the SAURON data, we have constructed the well-sampled 2D ionized-gas velocity field, but only for the very inner region, $R<8^{\prime \prime}(740 \mathrm{pc})$. In the meanwhile, due to the long 
TABLE 2

DETERMINATION OF THE GASEOUS DISK ORIENTATION.

\begin{tabular}{|c|c|c|c|c|c|c|}
\hline \# & Emission lines & Cross-sections & $i$ (deg) & PA (deg) & $\delta i_{1}$ & $\delta i_{2}$ \\
\hline 1 & all & all & 62 & 257 & $45 \pm 14$ & $89 \pm 15$ \\
\hline 2 & forbidden lines $[O I I I],[N I I],[S I I]$ & all & 47 & 261 & $34 \pm 12$ & $78 \pm 14$ \\
\hline 3 & Balmer lines $H \beta, H \alpha$ & all & 42 & 273 & $24 \pm 11$ & $77 \pm 15$ \\
\hline 4 & all & $P A_{\text {slit }}=220,268$ & 43 & 270 & $26 \pm 11$ & $77 \pm 14$ \\
\hline 5 & all & $P A_{\text {slit }}=313,358$ & 38 & 244 & $40 \pm 11$ & $64 \pm 13$ \\
\hline
\end{tabular}

exposures and to adaptive binning application, with the SCORPIO/long-slit we have derived emission-line velocities up to the edge of the stellar velocity measurements - toward $60^{\prime \prime}(5.6 \mathrm{kpc})$ from the center.

Close to the minor axis of the stellar disk $\left(P A=220^{\circ}\right)$, where the stellar rotation velocity projections are almost zero, the gas line-of-sight velocity oscillates with the amplitude of $50-70 \mathrm{~km} \mathrm{~s}^{-1}$. Inspecting the SAURON-based gas velocity field, we see a 'blue' velocity spot in $1^{\prime \prime}-2^{\prime \prime}$ (i.e. $90-190 \mathrm{pc}$ ) to the south from the nucleus; this peculiar region is to be discussed below, in the Subsection 5.3. Trying to reconcile the gas velocity profiles in four position angles, we come to the conclusion that the ionized-gas motions inside the central kiloparsec are quite different from those in the outer disk. Within $R<10^{\prime \prime}$ (900 pc) at least two mechanisms affecting the gas motions can be identified: the first, an active nucleus impact, and the second, non-circular velocities induced by the circumnuclear spiral wave which settles perhaps off the stellar disk plane. At the same time, the outer gas rotates rather regularly, though in other plane than the stars do.

We have performed a series of calculations of the circularly rotating model of a thin disk for the outer gas (at $R=25^{\prime \prime}-50^{\prime \prime}, 2.3-4.7 \mathrm{kpc}$ ) alternately excluding different cross-sections and emission lines, using the same formulae as described in the Section 4.1.

The considered configurations (combinations of the emission lines in different cross-sections) are listed in the Table 2. Fig. 5 shows the comparison of the model rotation curves (blue lines) with the observed line-of-sight velocities for every long-slit cross-section. The first configuration (the top row of the Fig. 5) involves all available cross-sections. It shows a good agreement with the data in $P A=220^{\circ}$ and $P A=268^{\circ}$, however it has a mismatch with the observed velocities in $\mathrm{W}-\mathrm{N}\left(P A=313^{\circ}\right)$ and $\mathrm{N}$ $\left(P A=358^{\circ}\right)$ sides of the galaxy disk. The main problem is significant differences of the velocities estimated in the $H \beta$ and $[O I I I]$ emission lines (see discussion below). From this reason we have also considered separately fitting for the forbidden ([OIII], [SII], [NII]) and Balmer $(H \alpha, H \beta)$ emission lines, see the corresponding configurations \#2 and \#3. Note that resulting rotation curve in the case \#2 seems to be more realistic comparing with an abrupt decrease of the rotation velocities for \#3. It may be related to the fact that some part of the Balmer emission is produced by the main disk (see below). Also we have considered the numerical stability of our fitting using different combinations of cross-sections. In the configuration $\# 4$ we excluded points along $P A=313,358^{\circ}$ with the most dramatic difference between the ionized gas kinematics in the forbidden and Balmer lines. On the contrary, in the configuration \#5 only points from the $P A=313,358^{\circ}$ cross-sections were used.

Further, we determine the angle between the stellar and gaseous disks using the following formula (Moiseev 2008):

$\cos \delta i=\cos \left(P A_{\text {gas }}-P A_{\text {star }}\right) \sin i_{\text {star }} \sin i_{\text {gas }}+\cos i_{\text {star }} \cos i_{\text {gas }}$.

Here we assume that $i<90^{\circ}$ if the angular momentum vector is directed towards the observer, while in the opposite momentum direction the inclination is $180^{\circ}-i$. Supposing that the large-scale spiral arms in the stellar disk are trailing, we determine $i_{\text {star }}=40^{\circ}$. However, for the gaseous disk we cannot choose between the alternate directions of its angular momentum vector, hence for the mutual inclination angle we obtain two possible solutions, $\delta i_{1}$ and $\delta i_{2}$. Table 2 presents the resulting parameters of different configurations.

The mutual inclination angles for all configurations considered here are in agreement with each other within the error bar. In any case (see the two last right columns of Table 2), the large-scale gaseous disk appears to be strongly inclined to the main symmetry plane of the galaxy. The mean values for these angles, averaged over all our experiments, are $\delta i_{1}=34 \pm 9^{\circ}$, or $\delta i_{2}=77 \pm 9^{\circ}$. Here the errors correspond to rms. We have adopted these values as the possible mutual inclination angle between the stellar and the gaseous disks.

Meanwhile, the difference between the rotation planes of the gas, emitting mostly in the forbidden lines (shockexcited gas) and that, emitting prominently in the Balmer lines (star-formation excited gas) may be real. Also, we think that the difference in the orientation parameters for the two groups of the cross-sections (the experiments number 4 and 5 in the Table 2) may be physically motivated: perhaps, to the south from the line-of-nodes at $P A_{\text {gas }}=220^{\circ}-270^{\circ}$, we mainly see the inclined, shock-excited gaseous disk, and to the north from the line-of-nodes, this inclined gaseous disk is seen through the own gas-poor disk of NGC 7743, possible illuminated by a small amount of young stars.

\subsection{Gas ionization sources}

In order to identify the source of gas ionization, we plot our measurements for the inner part of the galaxy, where several emission lines of various excitation degrees are measurable, onto the classical excitation-type diagnostic diagrams (Fig. 6). We do not need to take into account the intrinsic reddening as we use the intensity ratios of close pairs of emission lines. One can see (Fig. 6) that all the measurements are located in the AGN-ionization area of the diagrams. With the increasing distance from the center, the contribution of the non-thermal source into the gas excitation diminishes because the [OIII] $\lambda 5007$ intensity decreases and the observed points cross the border between the AGN-type and LINER-type excitation ([OIII] $/ \mathrm{H} \beta=3$ Veilleux \& Osterbrock 1987). The off-center measurements can be fitted best of all by recent models of shock excitation for the low-density gas 


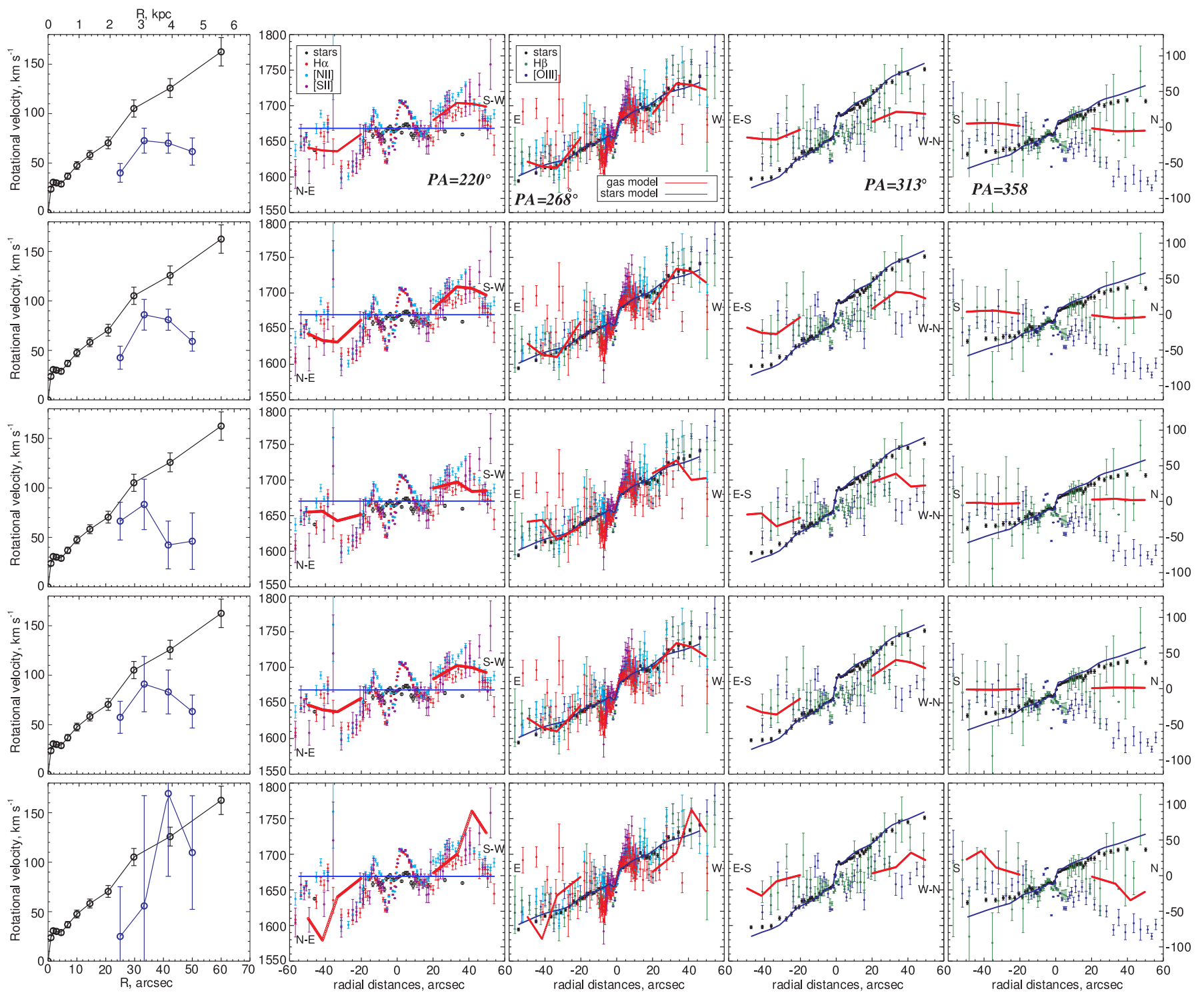

FIG. 5. - Series of experiments to determine the circularly rotating model of a thin disk to the outer gas. Each raw corresponds to the model configuration described in the Table 2- \#1 to 5 from top to bottom, respectively. The left column shown the stellar rotational model (black lines and circles) and the gaseous one (blue lines and circles). The following columns correspond to different cross-sections. Black circles present line-of-sight stellar velocity, color circles - gaseous velocity. The blue and red lines show the stellar and gaseous rotation model projections to different cross-sections, respectively.

(shock+precursor, Allen et al. 2008) if one supposes a moderate value of the magnetic parameter $B$ and the speed of the shock wave of more than $250 \mathrm{~km} \mathrm{~s}^{-1}$.

Therefore, while in the vicinity of the nucleus the gas ionization is mainly produced by an active non-thermal radiation source, just in 300-500 pc from the center the main contributors into the ionization are the shock waves. Perhaps, one of the local producer of the shock wave is the jet of the active nucleus (discussed below). For the more distant parts of the gaseous disk it is reasonable to relate shock waves to the inclined orbits of the gas clouds, which must collide with the interstellar medium of the main large-scale stellar disk (even if the density of the latter is very low). Moreover, a shock wave may be induced by a simple gas cloud, crossing the potential well of the stellar disk, quite analogous to the shock being induced by a gas cloud, crossing a spiral density wave in large-scale disks of spiral galaxies. This mecha- nism was discussed by Wakamatsu (1993), who considered the shock wave generation in the gas of polar ring galaxies. In favor of the latter hypothesis we can refer to the measurements along $P A=220^{\circ}$, where we trace the emission-line intensity ratios towards the largest distance from the center. At the radii of $R=20^{\prime \prime}-55^{\prime \prime}(1.9$ $5.1 \mathrm{kpc})$ we obtain $\log [\mathrm{NII}] / \mathrm{H} \alpha>-0.3$ that excludes photoionization by young stars (Stasińska et al. 2006). Therefore, throughout the whole large-scale gaseous disk of NGC 7743 the gas is ionized by shock waves.

\subsection{Jet-clouds interaction}

Fig. 2 2 shows that the velocity dispersion of ionized gas in the center of NGC 7743 exceeds that of the stellar component more than twice; evidently, it is an active nucleus effect. Moreover, in the long-slit cross-sections along $P A=313^{\circ}$ and $P A=358^{\circ}$ the gas velocity dispersion peaks are displaced from the (optical) nucleus 

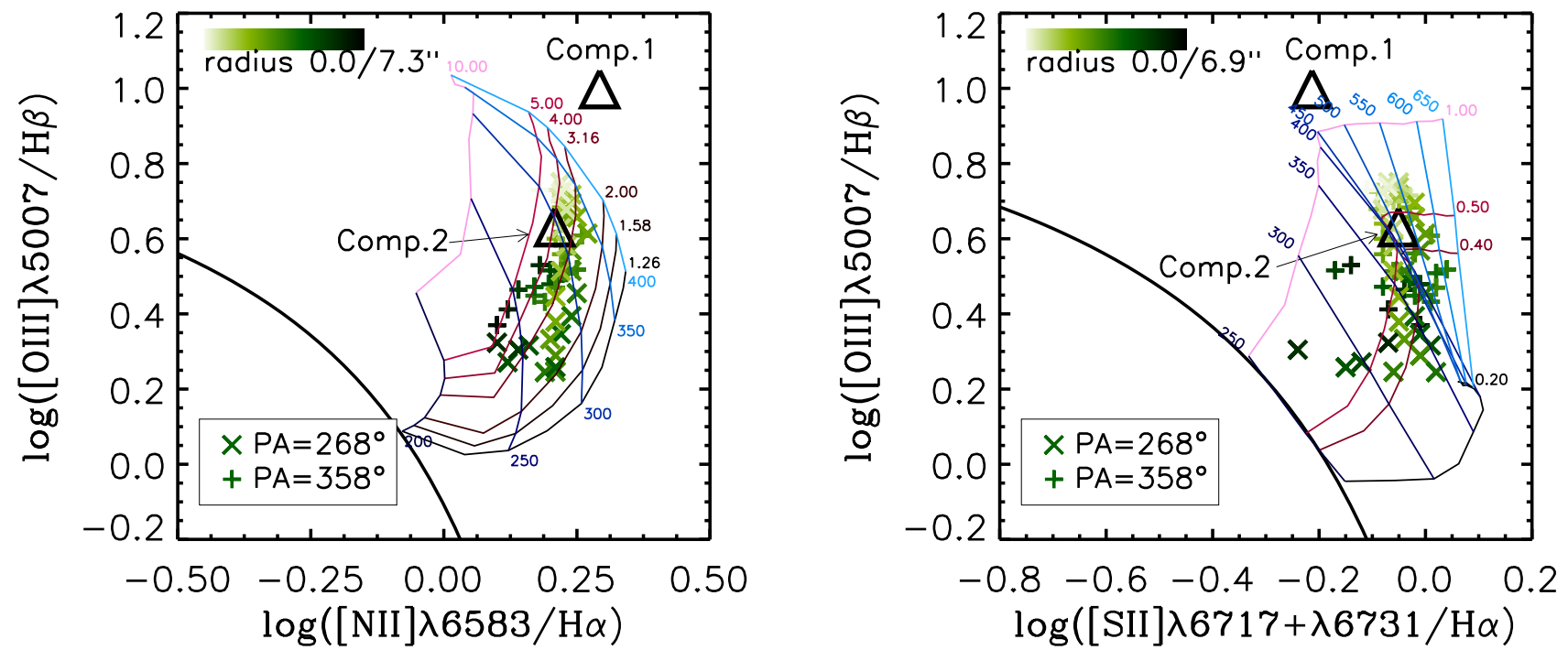

FIG. 6. - The excitation diagnostic diagrams confronting the emission-line intensity ratios: $[\mathrm{O}$ III $] / \mathrm{H} \beta$ vs $[\mathrm{N} \mathrm{II]}] / \mathrm{H} \alpha$ (left) and $[\mathrm{O}$ III] $/ \mathrm{H} \beta$ vs $[\mathrm{SII}] / \mathrm{H} \alpha$ (right). The pluses and crosses mark different long-slit cross-sections. The intensity of the green color corresponds to the increasing distance from the center in the sky plane. The solid black curve, which separates the areas with the AGN-induced and star formation induced excitations, is taken from Kewlev et al. (2001). Our measurements are overlaid by the shock-excitation model (Allen et al. 2008) for the electron density of $n_{e}=0.1 \mathrm{~cm}^{-3}$ and solar element abundances. The red colour traces magnetic parameter isolines, blue - the shock velocity isolines. Black triangles mark two separate emission-line components for the regions with clear emission-line splitting; 'comp.1' relates to the blueshifted component, 'comp.2' - to the main one.

by $1^{\prime \prime}-2^{\prime \prime}$ (i.e. by $90-190$ pc), and the gas line-of-sight velocity drops at this radius. The SAURON maps reveal this displacement even better: in Fig. 3 one can see a 'blue' velocity spot and the gas velocity dispersion peak at $2^{\prime \prime}$ to the south from the nucleus. Within this 'spot' the emission-line profiles in the SAURON spectra look asymmetric, and the SCORPIO spectra, which have better spectral resolution allow to decompose the entire profiles into two emission-line components (Fig. 7]). We simultaneously fitted the emission-line profiles for $\mathrm{H} \beta$, [O III], [N II], $\mathrm{H} \alpha$, and [S II] via a set of Gaussians broadened with the LSF, by requiring the same velocity difference between two components for all species. The best fit is presented in Fig. [7] the more intense component of all the lines (Comp. 2) corresponds to the regularly rotating gas, and the faint emission-line components (Comp. 1) are blueshifted by $299 \pm 8 \mathrm{~km} \mathrm{~s}^{-1}$. Big black triangles in Fig. 6] show intensity ratios for two components separately. One can see that the ratio of $[\mathrm{OIII}] / \mathrm{H} \beta$ for the off-nuclear Comp. 1 is higher than this ratio in the whole disk, and it is even higher than that in the close vicinity of the active nucleus. A similar picture - a high-speed strong shock excitation along with noncircular motions of the gas in a local off-centered area has been observed by Smirnova \& Moiseev (2010) in the Seyfert galaxy Mrk 334, where this area has been identified as a point of a satellite fragment crossing the galaxy disk. Another consequence of such crossing must be the lower gas density in this 'hole' with respect to the neighboring disk. However in NGC 7743 on the contrary, the blueshifted Comp. 1 reveals a very high electron density, $3000 \pm 1500 \mathrm{~cm}^{-3}$, which is determined from the intensity ratio of $[\mathrm{S} \mathrm{II}] \lambda 6717 /[\mathrm{S} \mathrm{II}] \lambda 6731$, while the regularly rotating disk Comp. 2 only has $n_{e}=150 \pm 30 \mathrm{~cm}^{-3}$. Therefore, in this peculiar region we detect gas compres- sion at the shock wave front. The probable cause of the shock waves can be an active-nucleus jet, penetrating the surrounding interstellar medium. Such intrusions are observed in many Seyfert galaxies, for example, in Mrk 3 (Capetti et al. 1999). Unfortunately, for NGC 7743 we have no narrow-filter emission-line images with a sufficiently good spatial resolution to inspect this peculiar region in detail. But Regan \& Mulchaey (1999) mentioned a 'blue-color excess' just in $1^{\prime \prime}(\sim 90 \mathrm{pc})$ to the SE from the nucleus at the HST map of the galaxy - it may be the region of the suggested jet intrusion. In this case, we only see the jet directed towards us, while the counterjet is obscured by the galaxy disk.

The radio image of the jet would be a decisive proof for this suggestion. But, as we noted in Section 2, all the radiocontinuum maps of NGC 7743 demonstrate only its compact core. Perhaps, the spatial resolutions and sensitivities of these radio observations are insufficient to resolve a jet.

\section{DISCUSSION}

The previously known data (see Section 2) characterized NGC 7743 as an ordinary early-type spiral galaxy with a weak nuclear activity. The traces of nuclear jet intrusion (the size of the jet must be less than 200 pc) into the interstellar medium, which we have found, are not so surprising for a Seyfert galaxy. However, another astonishing fact is revealed: the bulk of the ionized gas, up to the distance of $4-5 \mathrm{kpc}$ from the center, rotates on the orbits strongly inclined (by $\delta i=34^{\circ}$ or by $78^{\circ}$ ) to the plane of the stellar disk.

The large-scale disk of NGC 7743 lacks prominent HIIregions, and the gas excitation determined by us from the emission-line spectrum is shock-like. A similar situation has been observed by us in NGC 5631 (Sil'chenko et al. 
2009), where the large-scale gaseous disk is slightly inclined to the stellar one, and has an opposite spin. We have suggested two possible origins of the shock-like excitation: it can be either due to the collisions between the accreted gaseous clouds on the inclined orbits with the original low-density gaseous disk coplanar to the stellar one, or due to the accreted gas clouds, crossing the gravitational well of the stellar disk. However, in any case the gaseous content of the main galactic disk must be several times smaller than the accreted gas mass. Otherwise we would see two-component emission-line profiles over the whole disk extension, not only in the jet region.

Unfortunately, we have not found any simulations of shock waves induced in inclined gaseous disks in the literature, except the estimations by Wakamatsu (1993) for the case of polar rings. However, qualitatively we consider the mechanisms, proposed above as quite probable and similar to the spiral density wave generation in large-scale gaseous disks of spiral galaxies and to the shock-induced dust lanes at the leading edges of bars. These scenario can also explain the complex gas velocity behavior in the inner part of NGC 7743: the large-scale bar of the galaxy does not affect the gas at large distances from the center, but can affect its rotation at $R<20^{\prime \prime}$.

Among the galaxies known as 'polar-ring galaxies' we can note several cases of strongly inclined rings. First of all, it is NGC 660 where the outer gas-dust ring is inclined by $\delta i=63^{\circ}$ to the main galactic disk (Arnaboldi \& Galletta 1993). An inclined gaseous disk, less pronounced than that in NGC 660, and more similar to the case of NGC 7743 has been recently found in Arp 212 by Moiseev (2008). In Arp 212, a set of HIIregions within the radii of $2-6 \mathrm{kpc}$ concentrates in the plane, which is inclined by $\delta i=30^{\circ}-50^{\circ}$ with respect to the inner disk of the galaxy; in the regions where two disks are crossing, prominent shock fronts are seen. Moiseev (2008) suggested gas accretion from the neighboring gas-rich satellite, as the most probable mechanism of the inclined disk formation in Arp 212. Such a scenario for the formation of polar and inclined gaseous rings was firstly proposed by Reshetnikov \& Sotnikova (1997) and greatly developed during the past years, see Bournaud \& Combes (2003). We think that a similar scenario has provoked formation of the gaseous disk in NGC 7743. An alternate explanation may be minor merging or even a disruption of a gas-rich satellite by tidal forces from NGC 7743. But the latter scenario would produce other consequences, such as kinematically decoupled stellar subsystems, or the outer shells and arcs, which are not observed in NGC 7743. We hence conclude that in any case a possible contribution of the accreted stellar component in NGC 7743 would be small, and we have to come back to the gas-dominated accretion as the probable cause of the present state of NGC 7743 .

In Section 2 we have already mentioned that near NGC 7743 there are at least two gas-rich satellites, so there is no problem to identify a source of gas accretion. Moreover, in 50' (279 kpc) from NGC 7743, another S0-galaxy, NGC 7742, is located; the systemic velocity of NGC 7742 differs from that of NGC 7743 by only $50 \mathrm{~km} \mathrm{~s}^{-1}$. Let us imagine that earlier NGC 7742 and NGC 7743 approached each other with a typical rela- tive velocity of $200 \mathrm{kms}^{-1}$; then the time spent after this passage is about $1.4 \mathrm{Gyr}$. This time is comparable to the age of the nuclear star formation burst in NGC 7743, that may be induced by this interaction. However, we doubt that the inclined gaseous disk was formed during the same event, because this inclined-disk configuration is dynamically unstable and cannot persist its inclined rotation during several periods. It is probable that the inclined-orbit gas was accreted more recently.

\section{CONCLUSIONS}

Using the data of deep long-slit spectroscopy, obtained at the Russian 6-m telescope, and the archive data of the integral-field spectrograph SAURON, we have studied the stellar population properties and kinematics of the stellar and ionized-gas components in the early-type disk galaxy NGC 7743. We report the following results.

- All the ionized gas at radii of $1.5-5.4 \mathrm{kpc}$ is confined to the disk, inclined strongly to the main stellar disk of NGC 7743. The angle between two disks is estimated; two possible solutions are obtained, $34 \pm 9^{\circ}$ or $77 \pm 9^{\circ}$, depending on the mutual disk orientation in the space. The most probable origin of this inclined gaseous disk is the accretion from the gas-rich environment of NGC 7743. The main contributor into the gas excitation are the shock waves, induced probably by inclined-orbit gas clouds, crossing the main stellar disk.

- Complex motions, multi-component emission-line profiles, and strong shock excitation of the gas inside a compact region in $1^{\prime \prime}-2^{\prime \prime}$ to the south from the nucleus are interpreted by us as the signatures of active-nucleus jet interaction with the surrounding ambient medium.

- We confirm the recent, about 1 Gyr ago, intense star formation burst in the very center of NGC 7743, perhaps stimulated by the tidal interaction with the neighboring NGC 7742.

The inclined gaseous disk in NGC 7743 has one of the lowest contrasts among the structures of this kind, discovered in external galaxies. We think that deep spectroscopy of other gas-poor early-type disk galaxies would allow to find a lot of such inclined gaseous disks, formed by gas accretion from external sources.

This research is partly based on the data, obtained from the Isaac Newton Group Archive which is maintained as part of the CASU Astronomical Data Centre at the Institute of Astronomy, Cambridge. We have made use of the NASA/IPAC Extragalactic Database (NED), which is operated by the Jet Propulsion Laboratory, California Institute of Technology, under contract with the National Aeronautics and Space Administration. We acknowledge the usage of the HyperLeda database. This work was supported by the Russian Foundation for Basic Research (project no. 09-02-00870). AVM is also grateful to the 'Dynasty' Fund. The authors thank the anonymous referee for constructive advice that has helped us to improve the paper. 

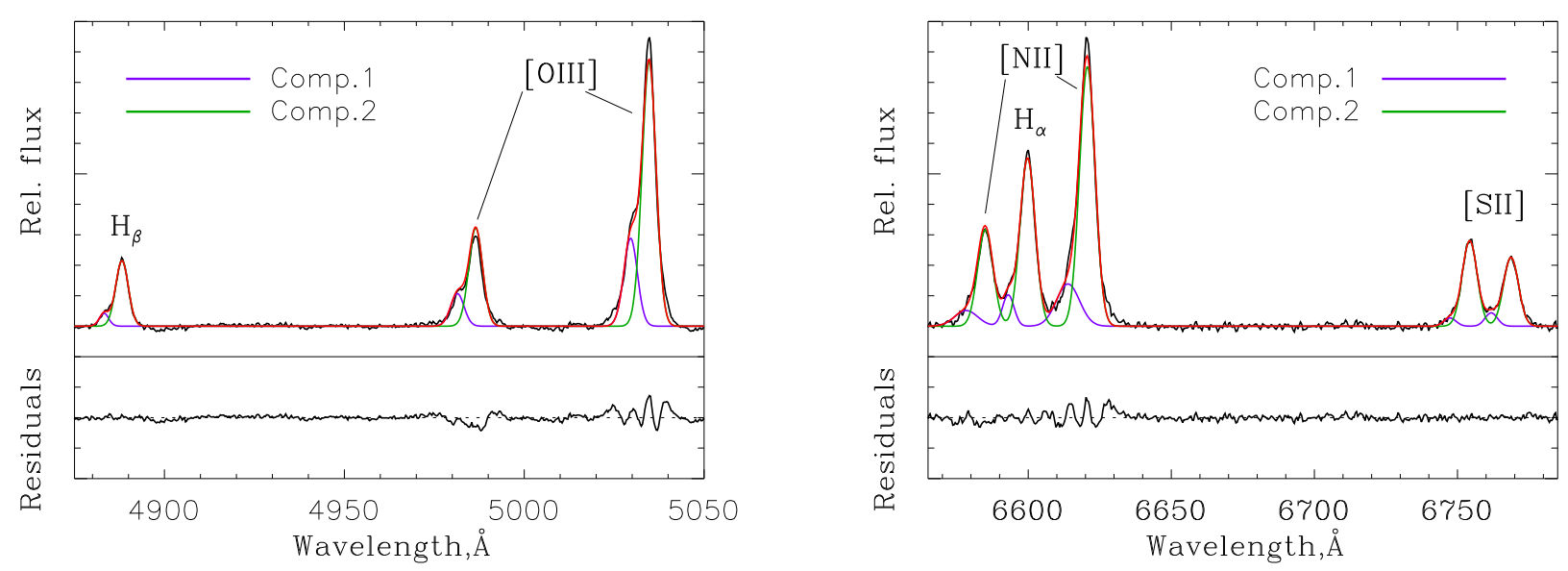

FIG. 7.- The multi-component emission-line profile, obtained by integrating the long-slit data within the range of $R=-4-0^{\prime \prime}$ along the cross-section in $P A=358^{\circ}$. The black line shows the observed emission-line spectrum, after subtracting the model of stellar spectra. The blue line corresponds to the Gaussian-profile model of the blueshifted component ('Comp. 1'), the green line - to the Gaussian-profile model of the main emission component ('Comp. 2'), and the red line traces the full model profile.

\section{REFERENCES}

Afanasiev, V. L.,\& Moiseev, A. V. 2005, Astronomy Letters, 31, 193

Afanasiev, V. L., Sil'chenko, O. K., Zasov, A. V. 1989, A\& A, 213, L9

Alonso-Herrero, A., Rieke, M. J., Rieke, G. H., Shields, J. C. 2000, ApJ, 530, 688

Allen, M .G., Groves, B .A., Dopita, M .A., Sutherland, R .S., Kewley, L .J. 2008, ApJS, 178, 20

Arnaboldi, M., Galletta, G. 1993, A\&A, 268, 411

Bacon, R., Copin, Y., Monnet, G. et al. 2001, MNRAS, 326, 23

Bournaud, F., Combes, F. 2003, A\&A, 401, 817

Capetti, A., Axon, D. J., Macchetto, F. D., Marconi, A., Winge, C. 1999, ApJ, 516, 187

Cappellari, M., Emsellem, E., Krajnovic, D. 2011, MNRAS, 413, 813

Coccato, L., Corsini, E. M., Pizzella, A., Bertola, F. 2007, A\&A, 465,777

Corsini, E. M., Pizzella, A., Coccato, L., Bertola, F. 2003, A\&A, 408, 873

Erwin, P. 2005, MNRAS, 364, 283

Erwin, P., Pohlen, M., Beckman, J. E. 2008, AJ, 135, 20

Duprie K., Schneider S. E. 1996, AJ, 112, 937

Ho, L. C., Filippenko, A. V., Sargent, W. L. W. 1997, ApJS, 112, 315

Ho, L. C., Ulvestad, J. S. 2001, ApJS, 133, 77

Jensen, J. B., Tonry, J. L., Barris, B. J. et al. 2003, ApJ, 583, 712

Katkov, I. Yu., \& Chilingarian, I. V., 2011, arXiv:1012.4125 [astro-ph.IM]

Kewley, L. J., Dopita, M. A., Sutherland, R. S., et al. 2001, ApJ, 556,121

Koleva, M., Prugniel, Ph., Bouchard, A., Wu, Y. 2009, A\&A, 501, 1269

Koleva, M., Prugniel, Ph., De Rijcke, S. 2008a, AN, 329, 968

Koleva, M., Prugniel, Ph., Ocvirk, P., Le Borgne, D., Soubiran, C. 2008b, MNRAS, 385, 1998

Kormendy, J., Kennicutt, R. C. 2004, ARA\&A, 42, 603
Laurikainen, E., Salo, H., Buta, R., Knapen, J. H. 2007, MNRAS, 381,401

Le Borgne, D., Rocca-Volmerange, B., Prugniel, P., Lançon, A.,

Fioc, M., Soubiran, C. 2004, A\&A, 425, 881

Maiolino, R., Ruiz, M., Rieke, G. H., Papadopoulos, P. 1997, ApJ, 485, 552

Martini, P., Regan, M. W., Mulchaey, J. S., Pogge, R. W. 2003, ApJS, 146, 353

Martínez-Delgado, D., Gabany, R. J., Crawford, K., et al. 2003, AJ, 140, 962

Moiseev, A. V., Valdés J. R., Chavushyan, V. H. 2004, A\&A, 421, 433

Moiseev, A.V. 2008, Astrophys. Bull., 63, 201

Nagar, N. M., Wilson, A. S., Mulchaey, J. S., Gallimore, J. F. 1999, ApJS, 120, 209

Regan, M.W., Mulchaey, J.S. 1999, AJ, 117, 2676

Reshetnikov, V., Sotnikova, N. 1997, A\&A, 325, 933

Sarzi, M., Falcón-Barroso, J., Davies, R. l., Bacon, R., Bureau,

M., et al. 2006, MNRAS, 366, 1551

Sil'chenko, O. K. 2006, ApJ, 641, 229

Sil'chenko, O. K., Moiseev, A. V., Afanasiev, V. L. 2009, ApJ, 694,1550

Sil'chenko, O. K., Chilingarian, I. V. 2011, Astronomy Letters, 37,3

Smirnova, A., Moiseev, A. 2010, MNRAS, 401, 307

Smirnova, A. A., Moiseev, A., Afanasiev, V. L. 2010, MNRAS, 408,400

Stasińska, G., Cid Fernandes, R., Mateus, A., Sodré L., Asari, N. V. 2006, MNRAS, 371, 972

Terashima, Y., Iyomoto, N., Ho, L. C., Ptak, A. F. 2002, ApJS, 139, 1

Veilleux, S., Osterbrock, D. E. 1987, ApJS, 63, 295

Wakamatsu, K. 1993, AJ, 105, 1745

Wozniak, H., Combes, F., Emsellem, E., Friedli, D. 2003, A\&A, 409, 469

Zasov, A.V., Moiseev, A.V., Khoperskov, A.V., Sidorova, E.A. 2008, Astronomy Reports, 52, 79 